\title{
Article \\ Correlation of Morphology Evolution with Carrier Dynamics in InN Films Heteroepitaxially Grown by MOMBE
}

\author{
Fang-I Lai ${ }^{1}$, Jui-Fu Yang ${ }^{2}$, Woei-Tyng Lin ${ }^{1}$, Wei-Chun Chen ${ }^{3} \mathbb{D}$, Yu-Chao Hsu ${ }^{4,5}$ (D) and Shou-Yi Kuo ${ }^{2,4, *(D)}$ \\ 1 Department of Electrical Engineering Program C, Yuan-Ze University, 135 Yuan-Tung Road, \\ Chung-Li 32003, Taiwan; filai@saturn.yzu.edu.tw (F.-I.L.); w.tyng.lin@gmail.com (W.-T.L.) \\ 2 Department of Electronic Engineering, Chang Gung University, 259 Wen-Hwa 1st Road, Kwei-Shan, \\ Taoyuan 33302, Taiwan; yoharecca@gmail.com \\ 3 Taiwan Instrument Research Institute, National Applied Research Laboratories, 20 R\&D Road V1, \\ Hsinchu Science Park, Hsinchu 30076, Taiwan; weichun@narlabs.org.tw \\ 4 Department of Urology, Chang Gung Memorial Hospital, Linkou, No. 5, Fuxing Street, Kwei-Shan, \\ Taoyuan 33305, Taiwan; hsuyc@cgmh.org.tw \\ 5 School of Medicine, Chang Gung University, 259 Wen-Hwa 1st Road, Kwei-Shan, Taoyuan 33302, Taiwan \\ * Correspondence: sykuo@mail.cgu.edu.tw; Tel.: +88-603-422-8800
}

Citation: Lai, F.-I; Yang, J.-F.; Lin, W.-T.; Chen, W.-C.; Hsu, Y.-C.; Kuo, S.-Y. Correlation of Morphology Evolution with Carrier Dynamics in InN Films Heteroepitaxially Grown by MOMBE. Catalysts 2021, 11, 886 . https://doi.org/10.3390/ catal11080886

Academic Editor: Leonarda

Francesca Liotta

Received: 18 May 2021

Accepted: 12 July 2021

Published: 22 July 2021

Publisher's Note: MDPI stays neutral with regard to jurisdictional claims in published maps and institutional affiliations.

Copyright: (c) 2021 by the authors. Licensee MDPI, Basel, Switzerland. This article is an open access article distributed under the terms and conditions of the Creative Commons Attribution (CC BY) license (https:// creativecommons.org/licenses/by/ $4.0 /)$.

\begin{abstract}
In this study, we report the catalyst-free growth of $n$-type wurtzite InN, along with its optical properties and carrier dynamics of different surface dimensionalities. The self-catalyzed epitaxial growth of InN nanorods grown by metal-organic molecular-beam epitaxy on $\mathrm{GaN} / \mathrm{Al}_{2} \mathrm{O}_{3}(0001)$ substrates has been demonstrated. The substrate temperature is dominant in controlling the growth of nanorods. A dramatic morphological change from 2D-like to 1D nanorods occurs with decreasing growth temperature. The InN nanorods have a low dislocation density and good crystalline quality, compared with InN films. In terms of optical properties, the nanorod structure exhibits strong recombination of Mahan excitons in luminescence, and an obvious spatial correlation effect in phonon dispersion. The downward band structure at the nanorod surface leads to the photon energy-dependent lifetime being upshifted to the high-energy side.
\end{abstract}

Keywords: InN; nanorods; catalyst-free

\section{Introduction}

Since Davydov et al. discovered the narrow band gap phenomenon of $\operatorname{InN}\left(E_{g} \sim 0.7 \mathrm{eV}\right)$, InN has attracted a wide range of research interest [1]. In addition, we expect InN to have the highest electron mobility among III-nitrides (approximately $4400 \mathrm{~cm}^{2} / \mathrm{V} \cdot \mathrm{S}$ ). These characteristics will enable InN to be considered a highly potential application material in many optoelectronic fields, such as high-speed electronic devices (HEMT), optoelectronic detectors, and photovoltaic and light-emitting devices [2-5]. However, due to a large number of external defects such as dislocation, defects, and so on, practical InN-based devices are still not achievable [6,7]. In addition, the Fermi energy level pinning above the conduction band is accompanied by a donor-like surface state, resulting in an ultrahigh density of the surface electron accumulation layer.

Most InN samples reported recently are $n$-type degenerate semiconductors, with an electron concentration of approximately $1 \times 10^{18} \mathrm{~cm}^{-3}$ or higher. Much research has been dedicated to understanding the fundamental properties of $\operatorname{InN}$, especially its photoluminescence (PL) properties [8-11]. The optical properties of semiconductors are very sensitive to the increase in carrier concentration and are reflected in the blueshift of peak energy and the expansion of the peak width. Many research teams have recently discussed the origin of the high emission peaks above the theoretical bandgap $(\sim 0.65 \mathrm{eV})$ in degenerate InN samples [11,12]. This high-energy peak has been ascribed to Mahan excitons and inspected by PL spectra and reflectivity measurement [12]. 
As III-V nitrides lack native substrates for these materials, there is a very large dislocation density in the film. As a result, the crystalline quality of these materials is reduced, which leads to a reduction in practical performance. As with other III-V nitrides, bulk $\mathrm{InN}$ also lacks a native substrate. Fortunately, the nanometer-scale quasi-one-dimensional (quasi-1D) structure has become a subject of intense research interest due to the low structural defect density $[13,14]$. This nanostructure shows promising potential for the realization of both fundamental physical research and the improvement of electronic, optoelectronic, and chemical/biological sensing properties with nanodevices. The morphology of quasi-1D InN, including nanorods, nanowires, nanotips, nanotubes and nanobelts, has been reported [15-17]. The intrinsic characteristic of electron accumulation at the InN surface has been considered for developing high-sensitivity vapor/liquid detection devices $[18,19]$. Several growth techniques have been used for the fabrication of InN nanostructures, with vapor-liquid-solid (VLS) catalytic growth from the bottom up being the most used method [20-22]. There is also the option of utilizing a catalyst for growth, but this requires a material that is liquefied and inert below the growth temperature.

Instead of a catalyst-assisted process, this study demonstrates a self-catalysis mechanism for the growth of InN nanorods by controlling the temperature through metal-organic molecular-beam epitaxy (MOMBE). The optical properties and carrier dynamics of $\mathrm{InN}$ samples, including film-type, nanorod-type, and mixed-type, were studied at different carrier concentrations (higher than $1 \times 10^{18} \mathrm{~cm}^{-3}$ ) using PL, time-resolved photoluminescence (TRPL), and Raman spectra. High-energy transitions appeared above the bandgap value of $0.7 \mathrm{eV}$, which was classified to the presence of Mahan excitons. We adopt the TRPL technique to further confirm the evidence of the presence of Mahan excitons, in which the spectra revealed a strong localization phenomenon near the Mahan excitons' energy.

\section{Results and Discussions}

Figure 1 shows the top-view and cross-sectional SEM images of InN materials grown at various temperatures, and the structure of $\mathrm{InN}$ transitioned from $1 \mathrm{D}$ nanorods to $2 \mathrm{D}$ thin films with increasing growth temperature. The length and diameter of the nanorods were determined to be approximately $1.05 \mu \mathrm{m}$ and $160 \mathrm{~nm}$, respectively. As the growth temperature increased, the surface became rougher with the coexistence of both nanorods and a film structure. The initial thickness of the film was approximately $580 \mathrm{~nm}$, but this increased to a maximum of $880 \mathrm{~nm}$ at the highest growth temperature. Table 1 lists detailed structural information of the InN samples.

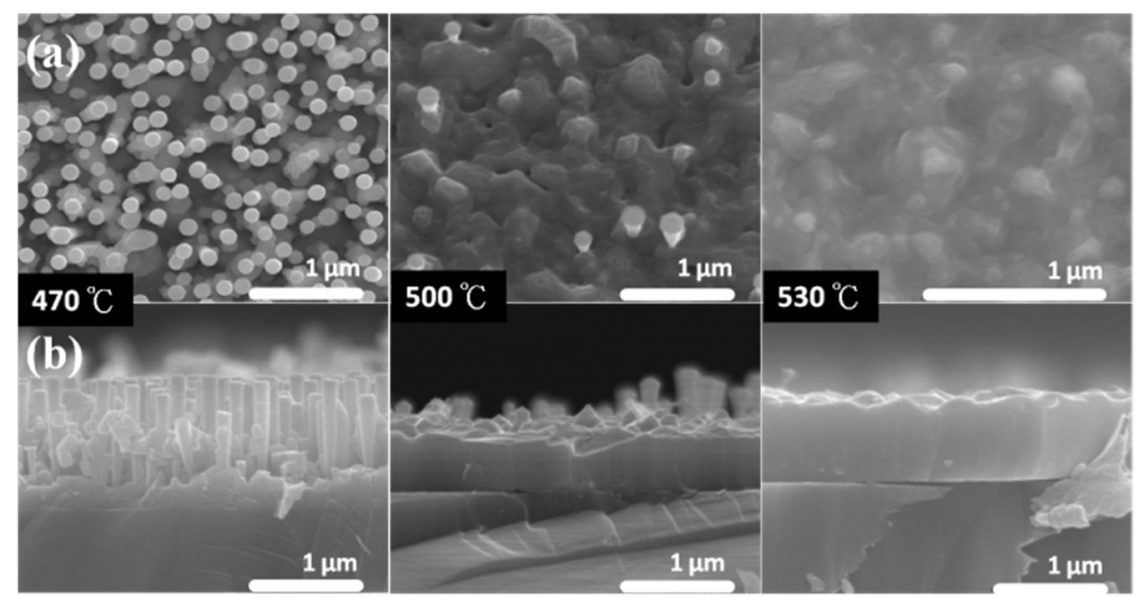

Figure 1. Scanning electron microscopy images of InN samples: (a) top-view images and (b) crosssectional images. 
Table 1. The dislocation density of all InN samples.

\begin{tabular}{ccccc}
\hline $\begin{array}{c}\mathbf{T}_{\mathbf{g}} \\
\left({ }^{\circ} \mathbf{C}\right)\end{array}$ & Morphology & $\begin{array}{c}\boldsymbol{D}_{\text {screw }} \\
\left(\mathbf{c m}^{-\mathbf{2}}\right)\end{array}$ & $\begin{array}{c}\boldsymbol{D}_{\text {edge }} \\
\left(\mathbf{c m}^{-2}\right)\end{array}$ & $\begin{array}{c}\boldsymbol{D}_{\text {dis }} \\
\left(\mathbf{c m}^{-2} \mathbf{)}\right.\end{array}$ \\
\hline 470 & Nanorods & $\sim 1.55 \times 10^{9}$ & $\sim 5.49 \times 10^{9}$ & $\sim 7.24 \times 10^{9}$ \\
\hline 500 & $\begin{array}{c}\text { Films and } \\
\text { Nanorods }\end{array}$ & $\sim 3.86 \times 10^{9}$ & $\sim 9.57 \times 10^{9}$ & $\sim 1.34 \times 10^{10}$ \\
\hline 530 & Films & $\sim 8.59 \times 10^{9}$ & $\sim 1.62 \times 10^{10}$ & $\sim 2.47 \times 10^{10}$ \\
\hline
\end{tabular}

As can be seen from our experimental results, the two-dimensional InN layer tends to form at higher growth temperatures, while 1D nanorods form at lower growth temperatures. In addition, the presence of metal droplets was not observed at the end of the 1D nanorod structure, which may elucidate the nanorods' growth through the self-catalytic process. In our experiment, the growth rate increased with the III/V flow ratio, which implies that the growth was in the N-rich regime, which is consistent with that reported by Yoshizawa et al. [23]. According to earlier literature, the growth conditions of the $\mathrm{N}$-rich regime can inhibit the formation of indium droplets; if no indium droplets appear, we may hypothesize that $\mathrm{InN}$ nanorods were grown through a no-catalytic-growth mechanism. Although no metal particles were observed at the top, we could not completely rule out the possibility of a VLS-like or self-catalyzed mechanism, as In atoms could desorb at high temperatures [24-27]. The pronounced 2D growth mode is related to the breakdown of anisotropic-directed growth at higher growth temperatures, and increased surface atomic mobility may cause more indium atoms to spread to the (0001) surface, providing more stable binding sites. At the lower temperature of $470{ }^{\circ} \mathrm{C}$, the InN nanorods (NRs) with nonpolar m-plane side facets grow spontaneously along the c-direction, due to the lower diffusion barrier of In adatoms along the m-plane than along the c-plane, as reported by Aliano et al. [25], which results in a higher sticking coefficient of In atoms on the c-plane than that on the m-plane. Thus, In adatoms have a low sticking coefficient on the m-plane InN NR sidewalls and nucleate on the c-terraces, promoting the 1D growth of dislocationfree InN NR and reaching a length of $1 \mu \mathrm{m}$ at a density of about $10^{9} \mathrm{~cm}^{-2}[25,26]$. A higher growth temperature of $\mathrm{InN}$ is expected to bring about a higher decomposition rate because of thermal decomposition of the grown InN. A pronounced 1D characteristic thereupon will appear for the $\mathrm{InN}$ grown at a lower temperature.

Figure 2a shows X-ray diffraction $\omega$-2 $2 \theta$ spectra for $\mathrm{InN}$ deposited directly on a GaN buffer layer, at various growth temperatures from $470^{\circ} \mathrm{C}$ to $530^{\circ} \mathrm{C}$. There are five diffraction peaks detected, corresponding to: $\operatorname{InN}(0002), \operatorname{InN}(0004)$ from wurtzite $\operatorname{InN}, \mathrm{GaN}(0002)$, $\mathrm{GaN}(0004)$ from wurtzite $\mathrm{GaN}$, and the (0006) diffraction from the sapphire substrate. However, no In metal peaks were detected in the patterns. These results indicate that $\mathrm{InN}$ films and nanorods with hexagonal structures are preferentially oriented and grown on a GaN template.

In order to determine the deposition relationship between $\mathrm{InN}$ and an underlying GaN template, the azimuthal $\omega$-scan of the $\operatorname{InN}(10 \overline{1} 2)$ and $\mathrm{GaN}(10 \overline{1} 2)$ diffraction peaks were adopted. The in-plane orientations of the $\operatorname{InN}(10 \overline{1} 2)$ are presented in Figure $2 b$. The hexagonal structure of InN produced six equally spaced peaks, without any other random peaks, suggesting that the InN grew predominantly in the [0001] direction. Despite this, the $0^{\circ}$ shift between $\mathrm{InN}$ and $\mathrm{GaN}$ reveals a coherent in-plane growth between the $\mathrm{InN}$ and $\mathrm{GaN}$ planes. These results indicate that $\mathrm{InN}$ films and nanorods were grown hetero-epitaxially on the GaN template with a hexagonal structure. 

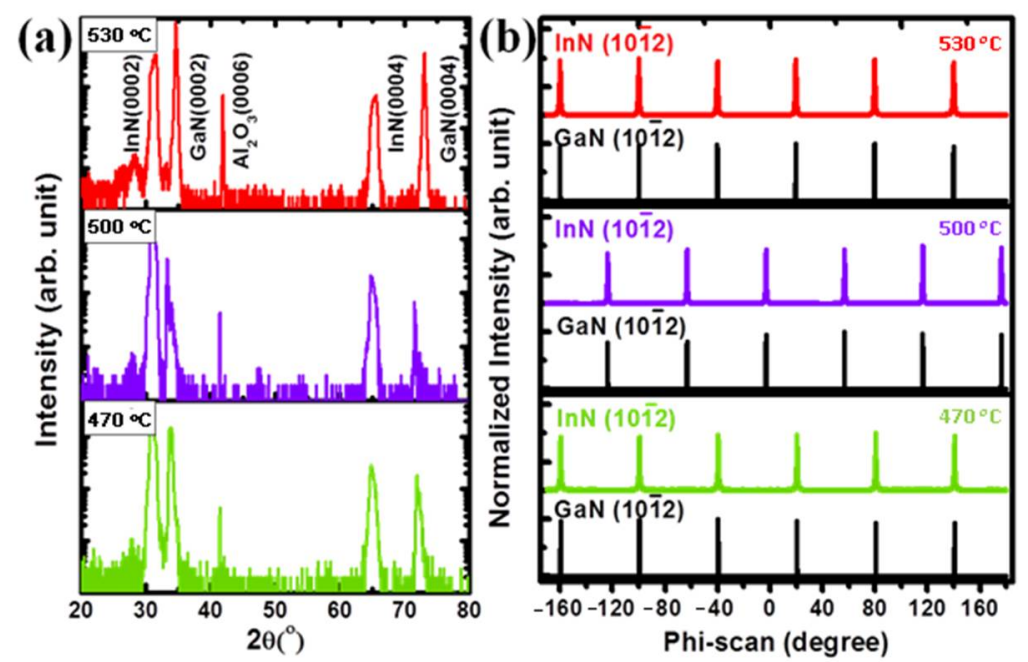

Figure 2. (a) XRD patterns of InN nanorods and films deposited on a GaN template. (b) Phi-scan patterns in XRD of InN(10̄12) phi-scan of nanorods and films.

The right axis of Figure 3 shows the c-axis lattice constant obtained from the InN films and nanorods that, utilizing the observed $\operatorname{InN}(0002)$ diffraction peak, is calculated to be larger than that of bulk $\mathrm{InN}(0.5703 \mathrm{~nm})$. The c-axis lattice constant of InN obviously shifts closer to the bulk value as the growth temperature is increased. This implies that the nanorods produced may be under strain, and this stain relaxed while the $\mathrm{InN}$ films formed.
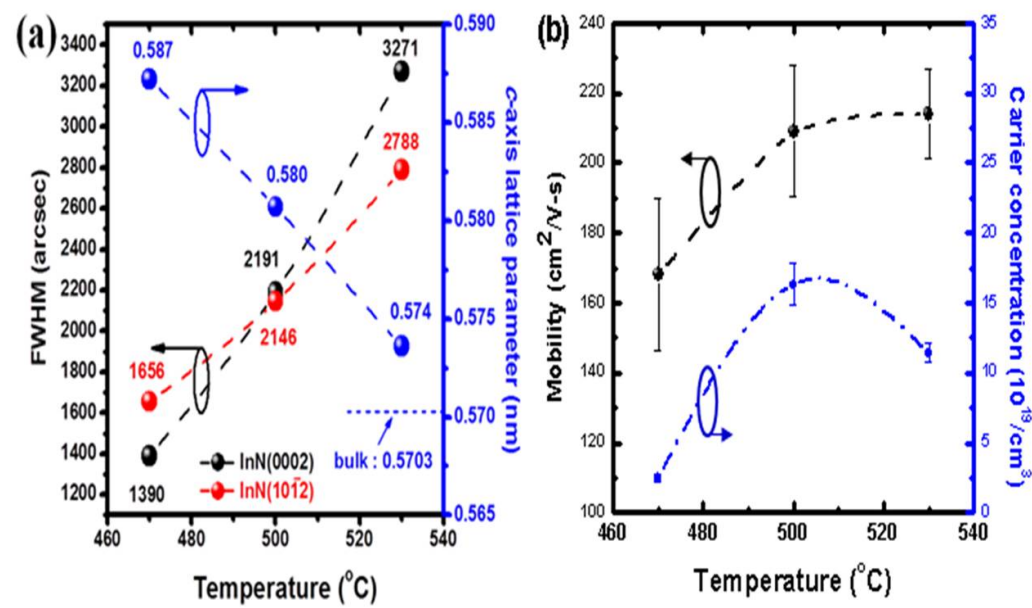

Figure 3. (a) FWHM of $\operatorname{InN}(0002)$ (black solid circle) and $\operatorname{InN}(10 \overline{1} 2)$ (red solid circle) and c-axis lattice parameter of the InN samples (blue solid circle). (b) Carrier concentration and mobility measured by Hall measurement.

Due to the lattice and thermal expansion coefficient mismatch between the $\mathrm{InN}$ and the substrate, dislocations form in epilayers to release the strain as the critical thickness is reached. The high density of dislocations in hetero-epitaxial epilayers is described by the mosaic block model. Specifically, the out-of-plane rotation perpendicular to the surface normal is the mosaic tilt, and the in-plane rotation around the surface normal is the mosaic twist.

$X R D$ rocking curves were measured for the $\operatorname{InN}(0002)$ and $\operatorname{InN}(10 \overline{1} 2)$ reflections using $\omega$-scanning geometry, in order to confirm the crystalline quality and calculate the density of dislocations in InN. The symmetric (0002)-reflecting plane is related to screw and mixed dislocations, and the asymmetric (1012)-reflecting plane is directly influenced by edge dislocations. As show in Figure 3a, the InN nanorods with a growth temperature of $470{ }^{\circ} \mathrm{C}$ have a greater crystalline quality in all $\mathrm{InN}$ samples, and the defects were apparently 
increased when the growth temperature and V/III ratio were increased. The following equation is used to estimate the dislocation density from the result of the XRD rocking curves [28-30]:

$$
D_{\text {dis }}=D_{\text {screw }}+D_{\text {edge }}=\frac{\beta_{(0002)}^{2}}{9 b^{2}}+\frac{\beta_{(10 \overline{1} 2)}^{2}}{9 b^{2}} \mathrm{~cm}^{-2}
$$

where $D_{\text {dis }}$ is the dislocation density, $D_{\text {screw }}$ is the compound dislocation density with screw and mixed dislocation, $D_{\text {edge }}$ is the compound dislocation density with edge and mixed dislocation, $\beta$ is the full width at half-maximum (FWHM) obtained from XRD rocking curves, and $b$ is the Burgers vector length. The calculated dislocation densities are shown in Table 1. In the InN nanorods obtained with a growth temperature of $470{ }^{\circ} \mathrm{C}$, the dislocation density was found to be $7.24 \times 10^{9} \mathrm{~cm}^{-2}$, or a third of that obtained with a growth temperature of $530^{\circ} \mathrm{C}$. In the InN films, the crystalline structure removes the mismatch in the lattice and thermal expansion coefficients through the formation of dislocations, resulting in a higher dislocation density than with nanorods. This phenomenon will be discussed in the following paragraph.

Figure $3 \mathrm{~b}$ shows the dependence of carrier concentration and mobility, as measured by the Hall effect. While the InN morphology changes from nanorods to films, the carrier concentration and mobility were found to be in the range $2.49 \times 10^{19} \mathrm{~cm}^{-3}, 1.63 \times 10^{20} \mathrm{~cm}^{-3}$, $1.14 \times 10^{20} \mathrm{~cm}^{-3}$; and 168, 209, $214 \mathrm{~cm}^{2} / \mathrm{V} \cdot \mathrm{s}$. Hall measurements were not performed on a single $\mathrm{InN}$ nanorod in this experiment, which was expected to yield a very high mobility and low carrier concentration. Direct determination of the carrier concentrations by Hall measurement on a single nanorod is not easy in practice. The electrical properties of our InN samples were measured using a Hall-effect measurement system at room temperature. In addition, the In pellet is used as an ohmic contact. The purity of the pellet is $99.99 \%$, and the diameter of the pellet is about $1 \mathrm{~mm}[13,14]$. The $\mathrm{InN}$ nanorods have a lower carrier concentration and mobility than the InN films, which is due to the comparatively greater crystalline quality and 1D structure of the InN nanorods. Fabrication of dislocation-free InN nanorods on heterogeneous substrates has been successfully investigated in the literature [26,31]. Therefore, we might expect only a little deviation from ideal stoichiometry of the InN nanorods, resulting in lower carrier concentration and higher mobility than InN films. However, the impact of surface roughness and ingrain and grain boundary scattering limits the electron mobility. Therefore, the results of Hall measurement on the InN samples are more suitable as an indication for the tendency, rather than an absolute value. Since the nearly dislocation-free $\mathrm{InN}$ nanorods are formed in our experiments, the difference between the defect characteristics of 1D nanorods and 2D thin-film, as listed in Table 1, will affect the electrical and optical properties of the InN material.

Figure 4a shows the low-temperature PL spectra of the InN samples, indicating that InN has dominant peaks with three kinds of recombination labeled as P1, P2, and P3. The emission spectrum exhibited a broadened feature with a higher carrier concentration, and the band-filling effect may dominate. This spectral feature is complex, and the emission peak is much greater than the accepted band gap $(0.65-0.7 \mathrm{eV})$. Those high-energy peaks are generally attributed to degenerate electrons-to-band or -to-acceptor level [31,32]. However, the peaks with high energy showed a stronger luminescence than those with low energy. This phenomenon is unlikely to be explained by degenerate-to-band, because of the lack of high $\mathrm{k}$-value holes in the valance band of degenerate $n$-InN. Therefore, the peaks with high energy could originate from degenerate electrons-to-acceptors.

In the early literature, it was reported that high emission energy and high-intensity luminescence were interpreted using Mahan excitons [33]. Mahan excitons can be described as localized holes of widespread $k$ that interact with electrons in the conduction band because the electrons are located on the Fermi edge. As the carrier scattering is enhanced, the optical transition matrix is extremely magnified, while the electrons below the Fermi energy level have only a weak recombination with the localized holes. Therefore, in the degenerate semiconductor, the high emission energy peaks show more intensive luminescence than that of the near-band edge types. 

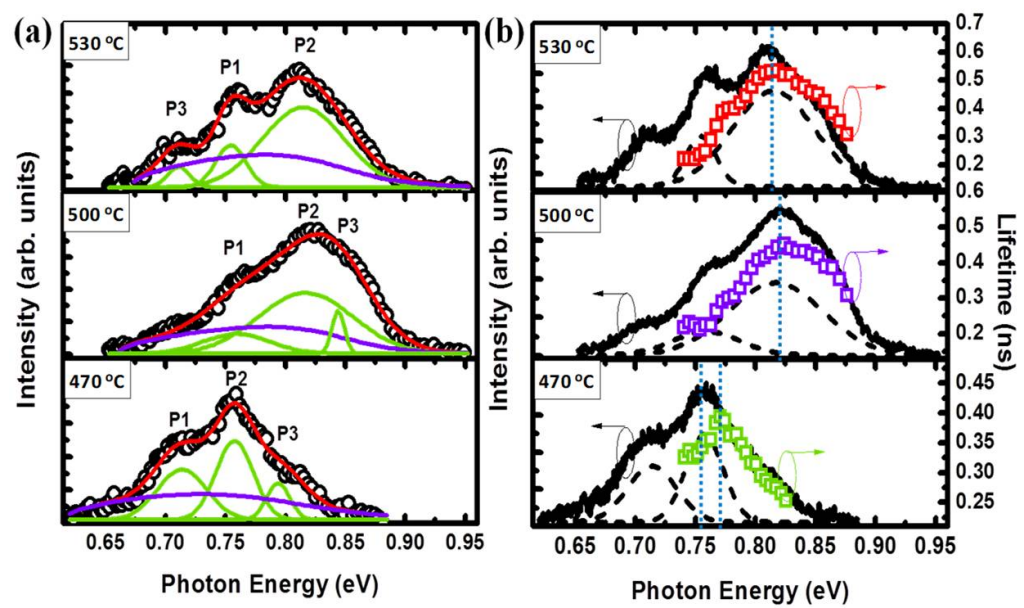

Figure 4. (a) Low-temperature photoluminescence spectra with fitting curve. (b) Energy-dependent TRPL and lifetime of the InN samples.

To further confirm the mechanism of carrier recombination, the low-temperature PL spectrum is fitted by an equation which assumes that the transition is due to free electron and acceptor recombination:

$$
I(\hbar \omega)=I_{0} \sqrt{\hbar \omega-\left(E_{g}-E_{A}\right)}\left(1+\exp \frac{\hbar \omega-E_{F}}{k_{b} T}\right)^{-1}
$$

In Equation (2), $\hbar \omega$ is the photon energy, $E_{g}$ represents the band gap value, $E_{A}$ is the energy of acceptor ionization energy, $E_{F}$ represents the quasi-Fermi energy, and $k_{b}$ and $T$ represent the Boltzman coefficient and electron temperature, respectively. During the fitting process, the Gaussian band was added to explain the enhanced effect of the Fermi edge singularity on the optical matrix of the Mahan excitons [34]. The fitting line shape is plotted in Figure 4a, which is in agreement with the experimental spectra, and a summary of the fitted results is provided in Table 2.

Table 2. Fitting results of the low-temperature PL spectra.

\begin{tabular}{cccccc}
\hline $\mathrm{T}_{\mathrm{g}}\left({ }^{\circ} \mathbf{C}\right)$ & $E_{\boldsymbol{g}}(\mathbf{e V})$ & $E_{F}(\mathrm{eV})$ & $E_{A}(\mathbf{m e V})$ & $E_{A 1}(\mathbf{m e V})$ & $E_{A 2}(\mathbf{m e V})$ \\
\hline 470 & 0.651 & 0.793 & 36 & 79 & 35 \\
500 & 0.698 & 0.842 & 38 & 83 & 25 \\
530 & 0.712 & 0.839 & 41 & 84 & 24 \\
\hline
\end{tabular}

These results show that two distinctly different acceptor levels participate in the recombination process (P1, P2 of all samples), and the Mahan exciton is represented by the strong luminescence intensity. According to the values obtained from fitting the results, the $\mathrm{P} 3$ of $\mathrm{T}_{\mathrm{g}}=470{ }^{\circ} \mathrm{C}$ and $500{ }^{\circ} \mathrm{C} \mathrm{InN} \mathrm{samples} \mathrm{is} \mathrm{attributed} \mathrm{to} \mathrm{the} \mathrm{free} \mathrm{electrons} \mathrm{at} \mathrm{the} \mathrm{Fermi}$ edge recombining with the free hole $(\mathrm{e}, \mathrm{h})$, and the $\mathrm{P} 3$ of $\mathrm{T}_{\mathrm{g}}=530^{\circ} \mathrm{C} \operatorname{InN}$ samples is related to the near-band-edge recombination (NBE). The band gap energy of an InN nanorod is $0.65 \mathrm{eV}$, which reveals a greater crystalline quality when compared to InN films $(0.70 \mathrm{eV}$, $0.71 \mathrm{eV})$. It is known that the structure and electronic properties of semiconductors can be altered by applying external stress or inducing internal strains through lattice and/or thermal mismatch strains in thin films [35-37]. Interatomic distances and the relative positions of atoms have a strong bearing on the band structure and $E_{g}$ in compound semiconductors. Previous research on III-nitride has pointed out that the wurtzite bandgap of III-nitride can be modulated by uniaxial and/or biaxial strains. Dong et al. and Duan et al. have proposed that the reduction in the band gap may be accomplished for modest and realistic strains $[35,36]$. As shown in Figure 3a of the manuscript, the degree of lattice relaxation is higher while increasing the growth temperature. Thus, the band gap energy 
of an InN nanorod may be slightly smaller than other InN films. Moreover, there is a small discrepancy between the theoretical line and experimental spectra at the lower energy side, which is attributable to the band tail states at the band edge that were neglected in Equation (2).

We used energy-dependent time-resolved photoluminescence (ED-TRPL) to further confirm the presence of Mahan excitons in these InN materials. Once Mahan excitons exist, the deep localized holes involved in the Mahan excitons can capture the carrier, preventing them from escaping from the localized states and prolonging lifetime. The results of the ED-TRPL in Figure $4 \mathrm{~b}$ show that a localized state does exist in the InN samples, in which lifetime can be fitted through the following single-exponential decay, such as Equation (3):

$$
I(t)=I_{0} \exp \left(-\frac{t}{\tau}\right)
$$

In Equation (3), $I_{0}$ represents luminescence intensity at $t=0$, and $\tau$ represents the carrier lifetime. The fitting results indicate that the peak position of longest lifetime coincides with the dominant peak (P2) for $\operatorname{InN}$ films $\left(\mathrm{T}_{\mathrm{g}}=500^{\circ} \mathrm{C}\right.$ and $\left.530{ }^{\circ} \mathrm{C}\right)$. Except the main peaks, the ED-TRPL data presented no significant change on the left side, which matched the second strongest peak (P1) for all samples. This standstill of lifetime also indicated a localization of the phenomenon related to Mahan excitons, but was weaker than the main peak because of the shorter carrier lifetime. However, the longest lifetime in the InN nanorods was located at a higher energy level than the main peak, which was assigned as near Mahan exciton recombination. This result might be because of the obvious bandbending phenomenon in the nanorod structure surface, resulting in the longest blue-shift lifetime compared with the peaks for Mahan exciton recombination.

A schematic of the carrier recombination in the InN samples is shown in Figure 5a. Transition 1 is Mahan exciton recombination with two different hole-localized acceptors with high k-values. Due to the widespread k of acceptor wave function, there was a strong luminescence intensity display due to the scattering rate being enhanced at the Fermi edge. Transitions 2 and 3 are Fermi-edge to valence-band recombination, and near-band-edge recombination with low k-value and $\Gamma$-point, respectively. The luminescence intensity of transitions 2 and 3 is weaker than transition 1 due to the small number of holes at the Fermi vector in the valence band and $\Gamma$-point.

Figure 5b shows the temperature-dependent PL spectra taken from samples with different structures. For the nanorod structure, the main peak shows a significant blue shift in Figure $5 \mathrm{c}$ at all temperature ranges. However, the PL signal decreased more rapidly than was seen with the film type samples. The spectrum cannot be analyzed at temperatures over $160 \mathrm{~K}$ due to the low S/N ratio. The phenomenon of blueshift in the main peak can be ascribed to the band bending near the nanorod's surface because of the electron accumulation layer. Compared with the film structure, the large surface-to-column ratio of the rod structure may cause a more significant band-bending phenomenon. The photoinduced holes tend to move from a low energy state at the rod center to a high energy state near the surface as the temperature increases, thus leading to an anomalous blue shift [38]. The rapid decrease in luminescence intensity in the InN nanorods can also be attributed to the large surface-to-column ratio, which makes the influence of surface state more significant. The InN films show a strong luminescent characteristic even at high temperatures. The position of the peak in Figure $5 c$ shows that there is no significant shift in any of the temperature ranges. In this case, the 2D film-type InN sample has a very high carrier concentration. In such a highly degenerate $n$-type InN semiconductor, the temperature-induced band gap shrinkage can be easily compensated by the increased electron Fermi level, so there is almost no peak shift [11]. 

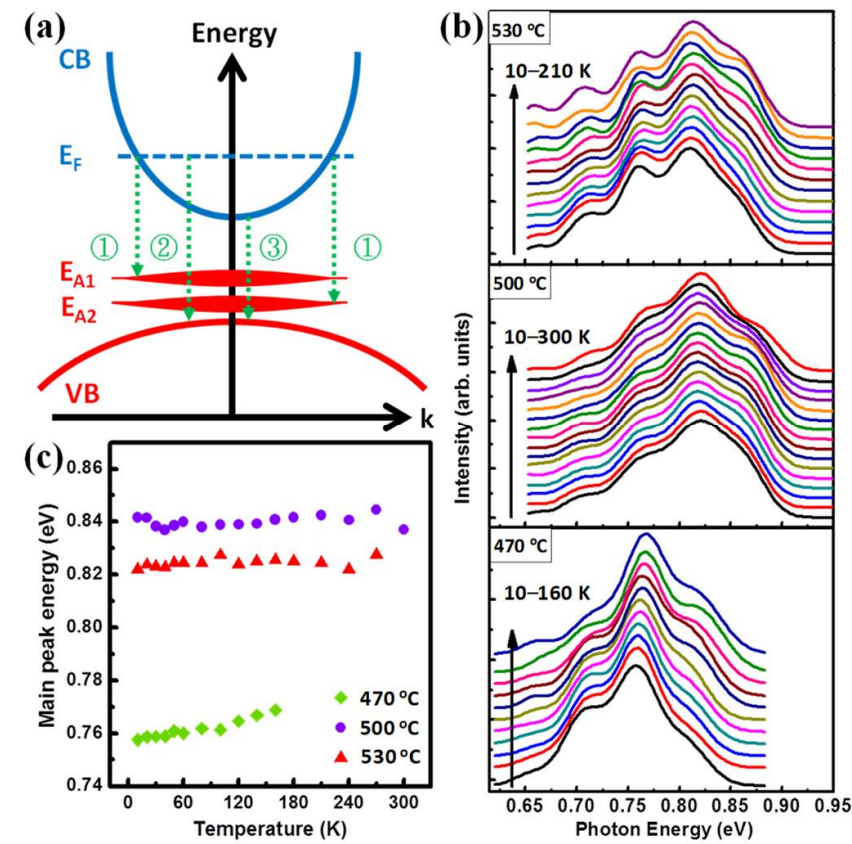

Figure 5. (a) Schematic of the recombination of an electron with hole in the InN samples. (1) Electron at Fermi edge recombines with localized hole at acceptor. (2) Electron recombines with hole at valence band. (3) Near-band-edge transition. (b) Temperature-dependent PL spectra of the InN samples. (c) The temperature-dependent main peak energy.

The hexagonal InN with a wurtzite structure was of the $\mathrm{C}_{6 \mathrm{v}}^{4}$ space group, including four atoms in a unit cell. For the factor group analysis at the $\Gamma$ point, theory predicts six optical modes as follows: $\Gamma_{\text {opt }}=A_{1}+2 B_{1}+E_{1}+2 E_{2}$. These six phonon branches all belong to a Raman active mode, with the exception of the silent $\mathrm{B}_{1}$ mode. In our roomtemperature Raman measurement, the $\mathrm{z}$ direction was found to be parallel to the c-axis and the $\mathrm{x}$ and $\mathrm{y}$ directions were normal to $\mathrm{z}$. According to the backscattering $\mathrm{z}(\mathrm{x},-)^{-} \mathrm{z}$ geometry, only the $\mathrm{E}_{2}$ and $\mathrm{A}_{1}(\mathrm{LO})$ modes are allowed by selection rules for the c-plane InN grown on the (0001) substrate. The Raman spectrum of the InN samples is displayed in Figure 6a. There are two well-resolved active phonon modes of $E_{2}$ (high) and $A_{1}(L O)$, which are located at approximately $491 \mathrm{~cm}^{-1}$ and $591 \mathrm{~cm}^{-1}$, respectively. The asymmetric line shape of the $\mathrm{A}_{1}(\mathrm{LO})$ modes can be described by Fano interferences [39], which occur between the zone-center LO phonons and the quasi-continuum electronic state along the c-axis. In the typical case of Fano interferences in Raman spectra, the peak of the $\mathrm{A}_{1}(\mathrm{LO})$ modes displays an asymmetrical broadening line width with an increased carrier concentration, due to the strong interaction between $\mathrm{A}_{1}(\mathrm{LO})$ phonons and the free carriers distributed in the in-plane of InN. It is worth noting that the $\mathrm{A}_{1}(\mathrm{LO})$ mode of $\mathrm{InN}$ nanorods exhibits a manifestly broad asymmetrical and frequency-downshifted line shape compared to the InN films, even though the carrier concentration of the InN nanorods is lower. This phenomenon implies that the $\mathrm{A}_{1}(\mathrm{LO})$ phonons are not solely influenced by carrier concentration.

Thus, the spatial correlation effect [40-42] is introduced to describe the expression of $A_{1}(\mathrm{LO})$ phonons for InN nanorods. The Raman intensity $I(\omega)$ at a frequency $\omega$ can be expressed by the following integral, and we assume a spherical correlation region and Brillouin zone for simplicity:

$$
I(\omega) \propto \int \exp \left(\frac{-q^{2} L^{2}}{4}\right) \times \frac{d^{3} q}{[\omega-\omega(q)]^{2}+\left(\Gamma_{0} / 2\right)^{2}}
$$

where $q$ is expressed in units of $2 \pi / a$, a is the lattice constant, $L$ is the spatial correlation length, $\omega(q)$ represents the phonon dispersion, and $\Gamma_{0}$ is the width of the intrinsic Raman line shape. A Gaussian spatial correlation function, $\exp \left(-2 r^{2} / L^{2}\right)$, leads upon Fourier 
transformation to an average over $q$ with a similar weighting factor $\exp \left(-q^{2} L^{2} / 4\right)$. This is used to account for $q$-vector relaxation related to finite-size effects, and structural disorder. For the relation of phonon dispersion, $\omega(q)$ is taken into consideration in a one-dimensional linear-chain model to approximately describe the dispersion:

$$
\omega^{2}(q)=A+\left[A^{2}-B[1-\cos (\pi q)]\right]^{1 / 2}
$$
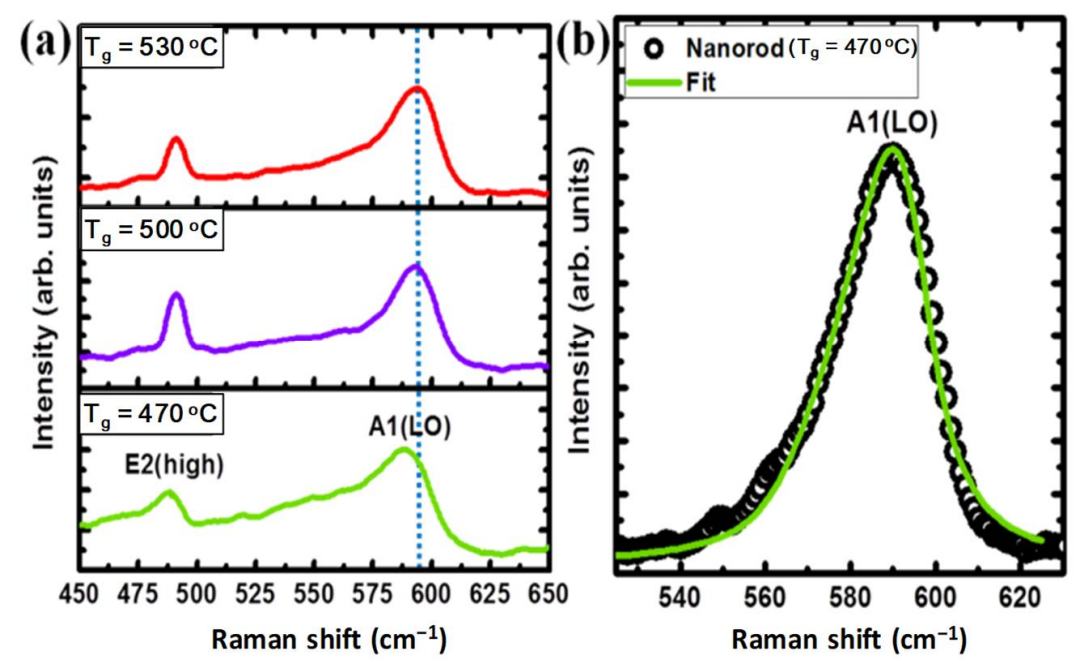

Figure 6. (a) Raman spectra with $\mathrm{E}_{2}$ (high) and $\mathrm{A}_{1}(\mathrm{LO})$ phonon modes. (b) The $\mathrm{A}_{1}(\mathrm{LO})$ phonon of nanorods with fitting curve by spatial correlation model.

Figure $6 \mathrm{~b}$ demonstrates that the fitted curve is in agreement with the experimental Raman line shape of the $\mathrm{A}_{1}(\mathrm{LO})$ mode. From the fitting results by Equations (4) and (5), we obtain the parameters: $A=1.77 \times 10^{5} \mathrm{~cm}^{-2}, B=5.86 \times 10^{9} \mathrm{~cm}^{-4}, \Gamma_{0}=15 \mathrm{~cm}^{-1}$, and $L=5 \mathrm{~nm}$. This clearly shows that the phonons in nanorods are influenced by the spatial correlation effect. The asymmetric broadening of the Raman line width of the $\mathrm{A}_{1}(\mathrm{LO})$ phonon may be caused by the following: (i) disorders in the crystalline structure induce the mode correlation functions of the phonon to become finite, with the relaxation of the $q=0$ selection rule associated with finite correlation length; (ii) the phonons at the Brillouin zone edge participate in inelastic collision with photons. The asymmetric broadening at the low energy side and downshifted frequency of the Raman line shape is due to the energy of the Brillouin zone edge being lower than that of the Brillouin zone center.

\section{Materials and Methods}

The samples of wurtzite InN were grown by metal-organic molecular-beam epitaxy (MOMBE, TIRI, Hsinchu, Taiwan) on c-plane sapphire substrates, and pre-growth of a GaN buffer layer by metal-organic chemical-vapor deposition (MOCVD, Aixtron, Herzogenrath, Germany). Group-III and N sources were trimethylindium (TMIn) and radio-frequency (RF) plasma-induced nitrogen atoms, respectively. The V/III flux ratio was fixed at approximately 1 , and the growth temperatures $(\mathrm{Tg})$ were $470{ }^{\circ} \mathrm{C}, 500{ }^{\circ} \mathrm{C}$, and $530{ }^{\circ} \mathrm{C}$. After preparing the InN samples, the morphology of each sample was observed with a scanning electron microscope (SEM, JEOL JSM-7000f, JEOL Ltd., Tokyo, Japan) operated at $10 \mathrm{kV}$, and the carrier concentration was measured by standard Hall-effect measurements at room temperature. Optical properties were determined by Raman spectroscopy, photoluminescence (PL) and time-resolved photoluminescence (TRPL). The Raman spectra was obtained by excitation with a He-Ne laser using $17 \mathrm{~mW}$ at $632.8 \mathrm{~nm}$, and an LN-cooled CCD detector was used to collect the backscattered light. In the PL measurement, the InN sample is placed in a temperature-controlled closed-cycle helium cryostat chamber. A semiconductor laser with a wavelength of $635 \mathrm{~nm}$ (PicoQuant LDH-D-C-635M dual 
mode laser, PicoQuant, Berlin, Germany) was used for PL and TRPL measurements, and it could be switched between continuous and pulsed mode. It was used to measure the TRPL repetition rate of $80 \mathrm{MHz}$, and the laser pulse width is 76 picoseconds. The PL and TRPL signals were dispersed by a monochromator (Jobin Yvon iHR 550, Taipei, Taiwan) using a 600 groove/mm grating and detected by an InGaAs photodiode (working range: 900-2100 nm) (Horiba, Taipei, Taiwan) and IR-PMT (working range 1000-1700 nm) (Horiba, Taipei, Taiwan), respectively.

\section{Conclusions}

This research reports the preparation of InN samples grown at different growth temperature by MOMBE, including nanorods and films. The structural and electronic properties shown in XRD patterns and Hall measurements reveal that the growth temperature is crucial in controlling the surface morphology of the InN films. A dramatic morphological change from 2D-like to 1D nanorods occurs with decreasing growth temperature. Compared with the InN films, the self-catalyzed $\mathrm{InN}$ nanorods have a low dislocation density and good crystalline quality. We observed and confirmed the presence of Mahan excitons from ED-TRPL, and temperature-dependent PL measurements. The spatial correlation model is introduced to show the phonon dispersion behavior in the nanorods. Experimental results show that the morphology of the InN samples can be controlled by the growth temperature, and that Mahan excitons exist in 2D film samples but not in 1D nanorod samples.

Author Contributions: Conceptualization: F.-I.L., W.-C.C. and S.-Y.K.; methodology, J.-F.Y. and Y.-C.H.; validation, F.-I.L., W.-T.L., S.-Y.K. and J.-F.Y.; formal analysis, W.-T.L. and Y.-C.H.; investigation, J.-F.Y., W.-T.L., W.-C.C. and Y.-C.H.; resources, F.-I.L., S.-Y.K. and Y.-C.H.; data curation, J.-F.Y. and W.-C.C.; writing-original draft, W.-T.L. and J.-F.Y.; writing-review and editing, W.-C.C. and S.-Y.K.; supervision, S.-Y.K.; project administration, F.-I.L.; funding acquisition, F.-I.L., S.-Y.K. and Y.-C.H. All authors have read and agreed to the published version of the manuscript.

Funding: This research was funded by Chang Gung Memorial Hospital (BMRP 956) and Ministry of Science and Technology (MOST 108-2112-M-182-001-MY3, MOST 107-2221-E-155-055-MY3).

Data Availability Statement: Data is contained within the article.

Conflicts of Interest: The authors declare no conflict of interest.

\section{References}

1. Arnaudov, B.; Paskova, T.; Paskov, P.; Magnusson, B.; Valcheva, E.; Monemar, B.; Lu, H.; Schaff, W.J.; Amano, H.; Akasaki, I. Energy position of near-band-edge emission spectra of InN epitaxial layers with different doping levels. Phys. Rev. B 2004, 69, 115216. [CrossRef]

2. Wang, T.; Wang, X.; Chen, Z.; Sun, X.; Wang, P.; Zheng, X.; Rong, X.; Yang, L.; Guo, W.; Wang, D.; et al. High-Mobility Two-Dimensional Electron Gas at InGaN/InN Heterointerface Grown by Molecular Beam Epitaxy. Adv. Sci. 2018, 5, 1800844. [CrossRef]

3. Lai, W.-J.; Li, S.-S.; Lin, C.-C.; Kuo, C.-C.; Chen, C.-W.; Chen, K.-H.; Chen, L.-C. Near infrared photodetector based on polymer and indium nitride nanorod organic/inorganic hybrids. Scr. Mater. 2010, 63, 653-656. [CrossRef]

4. Ataser, T The performance analysis of the GaAs/c-InN solar photovoltaic cell hetero-structure: Temperature dependence. Opt. Quantum Electron. 2020, 52, 407. [CrossRef]

5. Lin, W.; Li, S.; Kang, J. Near-ultraviolet light emitting diodes using strained ultrathin InN/GaN quantum well grown by metal organic vapor phase epitaxy. Appl. Phys. Lett. 2010, 96, 101115. [CrossRef]

6. Kalesaki, E.; Kioseoglou, J.; Lymperakis, L.; Komninou, P.; Karakostas, T. Effect of edge threading dislocations on the electronic structure of InN. Appl. Phys. Lett. 2011, 98, 072103. [CrossRef]

7. Stampfl, C.; Van de Walle, C.G.; Vogel, D.; Krüger, P.; Pollmann, J. Native defects and impurities in InN: First-principles studies using the local-density approximation and self-interaction and relaxation-corrected. Phys. Rev. B 2000, 61, R7846. [CrossRef]

8. Clinton, E.A.; Vadiee, E.; Tellekamp, M.B.; Doolittle, W.A. Observation and mitigation of RF-plasma-induced damage to III-nitrides grown by molecular beam epitaxy. J. Appl. Phys. 2019, 126, 015705. [CrossRef]

9. $\quad$ Fu, S.P.; Yu, C.J.; Chen, T.T.; Hsu, G.M.; Chen, M.J.; Chen, L.-C.; Chen, K.-H.; Chen, Y.F. Anomalous Optical Properties of InN Nanobelts: Evidence of Surface Band Bending and Photoelastic Effects. Adv. Mater. 2007, 19, 4524-4529. [CrossRef] 
10. Moret, M.; Ruffenach, S.; Briot, O.; Gil, B. The determination of the bulk residual doping in indium nitride films using photoluminescence. Appl. Phys. Lett. 2009, 95, 031910. [CrossRef]

11. Wei, P.C.; Chattopadhyay, S.; Lin, F.S.; Hsu, C.M.; Jou, S.; Chen, T., Jr.; Huang, P.-J.; Hsu, H.-C.; Shih, H.-C.; Chen, K.-H.; et al. Origin of the anomalous temperature evolution of photoluminescence peak energy in degenerate InN nanocolumns. Opt. Express 2009, 17, 11690-11697. [CrossRef] [PubMed]

12. Feneberg, M.; Däubler, J.; Thonke, K.; Sauer, R.; Schley, P.; Goldhahn, R. Mahan excitons in de-generate wurtzite InN: Photoluminescence spectroscopy and reflectivity measurements. Phys. Rev. B 2008, 77, 245207. [CrossRef]

13. Lebedev, V.; Cimalla, V.; Baumann, T.; Ambacher, O.; Morales, F.M.; Lozano, J.G.; Gonzalez, D. Effect of dislocations on electrical and electron transport properties of InN thin films. II. Density and mobility of the carriers. J. Appl. Phys. 2006, 100, 094903. [CrossRef]

14. Adikimenakis, A.; Chatzopoulou, P.; Dimitrakopulos, G.P.; Kehagias, T.; Tsagaraki, K.; Androulidaki, M.; Doundoulakis, G.; Kuzmik, J.; Georgakilas, A. Correlation of Threading Dislocations with the Electron Concentration and Mobility in InN Heteroepitaxial Layers Grown by MBE. ECS J. Solid State Sci. Technol. 2019, 9, 015006. [CrossRef]

15. Liang, C.H.; Chen, L.-C.; Hwang, J.S.; Chen, K.-H.; Hung, Y.T.; Chen, Y.F. Selective-area growth of indium nitride nanowires on gold-patterned Si(100) substrates. Appl. Phys. Lett. 2002, 81, 22-24. [CrossRef]

16. Lan, Z.; Wang, W.; Sun, C.; Shi, S.-C.; Hsu, C.-W.; Chen, T.; Chen, K.-H.; Chen, C.; Chen, Y.; Chen, L. Growth mechanism, structure and IR photoluminescence studies of indium nitride nanorods. J. Cryst. Growth 2004, 269, 87-94. [CrossRef]

17. Song, W.; Si, J.; Wu, S.; Hu, Z.; Long, L.; Li, T.; Gao, X.; Zhang, L.; Zhu, W.; Wang, L. Synthesis and morphology evolution of indium nitride ( $\mathrm{InN}$ ) nanotubes and nanobelts by chemical vapor deposition. CrystEngComm 2019, 21, 5356-5362. [CrossRef]

18. Lu, H.; Schaff, W.J.; Eastman, L.F. Surface chemical modification of InN for sensor applications. J. Appl. Phys. 2004, 96, 3577-3579. [CrossRef]

19. Kryliouk, O.; Park, H.J.; Wang, H.T.; Kang, B.S.; Anderson, T.J.; Ren, F.; Pearton, S.J. Pt-coated InN nanorods for selective detection of hydrogen at room temperature. J. Vac. Sci. Technol. B Microelectron. Nanometer Struct. 2005, 23, 1891-1894. [CrossRef]

20. Hsu, C.-W.; Ganguly, A.; Liang, C.-H.; Hung, Y.-T.; Wu, C.-T.; Hsu, G.-M.; Chen, Y.-F.; Chen, C.-C.; Chen, K.-H.; Chen, L.-C. Enhanced Emission of (In, Ga) Nitride Nanowires Embedded with Self-Assembled Quantum Dots. Adv. Funct. Mater. 2008, 18 , 938-942. [CrossRef]

21. Duan, X.; Lieber, C.M. Laser-Assisted Catalytic Growth of Single Crystal GaN Nanowires. J. Am. Chem. Soc. 2000, 122, 188-189. [CrossRef]

22. Zhang, J.; Zhang, L. Growth of semiconductor gallium nitride nanowires with different catalysts. J. Vac. Sci. Technol. B Microelectron. Nanometer Struct. 2003, 21, 2415-2419. [CrossRef]

23. Yoshizawa, M.; Kikuchi, A.; Fujita, N.; Kushi, K.; Sasamoto, H.; Kishino, K. Self-organization of GaN/Al0.18Ga0.82N multi-layer nano-columns on (0001) Al2O3 by RF molecular beam epitaxy for fabricating GaN quantum disks. J. Cryst. Growth 1998, 189, 138-141. [CrossRef]

24. Cheng, G.; Stern, E.; Turner-Evans, D.; Reed, M.A. Electronic properties of InN nanowires. Appl. Phys. Lett. 2005, 87, 253103. [CrossRef]

25. Aliano, A.; Catellani, A.; Cicero, G. Adatom kinetics on nonpolar InN surfaces: Implications for one-dimensional nanostructures growth. Appl. Phys. Lett. 2011, 99, 193106. [CrossRef]

26. Tangi, M.; De, A.; Ghatak, J.; Shivaprasad, S.M. Electron mobility of self-assembled and dislocation free InN nanorods grown on GaN nano wall network template. J. Appl. Phys. 2016, 119, 205701. [CrossRef]

27. Bertness, K.; Roshko, A.; Mansfield, L.; Harvey, T.; Sanford, N. Mechanism for spontaneous growth of GaN nanowires with molecular beam epitaxy. J. Cryst. Growth 2008, 310, 3154-3158. [CrossRef]

28. Gay, P.; Hirsch, P.; Kelly, A. The estimation of dislocation densities in metals from X-ray data. Acta Met. 1953, 1, 315-319. [CrossRef]

29. Ide, T.; Shimizu, M.; Shen, X.Q.; Jeganathan, K.; Okumura, H.; Nemoto, T. Improvement of film quality using Si-doping in Al-GaN/GaN heterostructure grown by plasma-assisted molecular beam epitaxy. J. Crystal Growth 2002, 245, 15-20. [CrossRef]

30. Gallinat, C.S.; Koblmüller, G.; Wu, F.; Speck, J.S. Evaluation of threading dislocation densities in In- and N-face InN. J. Appl. Phys. 2010, 107, 053517. [CrossRef]

31. Chang, Y.-L.; Li, F.; Fatehi, A.; Mi, Z. Molecular beam epitaxial growth and characterization of non-tapered InN nanowires on Si(111). Nanotechnology 2009, 20, 345203. [CrossRef]

32. Klochikhin, A.A.; Yu, V.; Davydov, V.Y.; Emtsev, A.V.; Sakharov, A.V.; Kapitonov, V.A.; Andreev, B.A.; Lu, H.; Schaff, W.J. Acceptor states in the photoluminescence spectra of $\mathrm{n}-\mathrm{InN}$. Phys. Rev. B 2005, 71, 195207. [CrossRef]

33. Mahan, G.D. Excitons in Degenerate Semiconductors. Phys. Rev. 1967, 153, 882-889. [CrossRef]

34. Feneberg, M.; Däubler, J.; Thonke, K.; Sauer, R.; Schley, P.; Goldhahn, R. Near band-gap photoluminescence of InN due to Mahan excitons. Phys. Status Solidi C 2009, 6, S385-S388. [CrossRef]

35. Dong, L.; Yadav, S.; Ramprasad, R.; Alpay, S.P. Band gap tuning in GaN through equibiaxial in-plane strains. Appl. Phys. Lett. 2010, 96, 202106. [CrossRef]

36. Duan, Y.; Qin, L.; Shi, L.; Tang, G.; Shi, H. Phase transition and band-structure tuning in InN through uniaxial and biaxial strains. J. Physics Condens. Matter 2013, 26, 025501. [CrossRef] 
37. Bhattacharyya, J.; Ghosh, S. Electronic band structure of wurtzite InN around the fundamental gap in the presence of biaxial strain. Phys. Status Solidi A 2007, 204, 439-446. [CrossRef]

38. Shen, C.-H.; Chen, H.-Y.; Lin, H.-W.; Gwo, S.; Klochikhin, A.A.; Davydov, V.Y. Near-infrared photoluminescence from vertical InN nanorod arrays grown on silicon: Effects of surface electron accumulation layer. Appl. Phys. Lett. 2006, 88, 253104. [CrossRef]

39. Inushima, T.; Higashiwaki, M.; Matsui, T. Optical properties of Si-doped InN grown on sapphire (0001). Phys. Rev. B 2003, 68, 235204. [CrossRef]

40. Cheng, C.L.; Chen, Y.F.; Chen, R.S.; Huang, Y.S. Raman scattering and field-emission properties of RuO2 nanorods. Appl. Phys. Lett. 2005, 86, 103104. [CrossRef]

41. Tiong, K.K.; Amirtharaj, P.M.; Pollak, F.H.; Aspnes, D.E. Effects of As+ ion implantation on the Raman spectra of GaAs: “Spatial correlation" interpretation. Appl. Phys. Lett. 1984, 44, 122-124. [CrossRef]

42. Wang, J.; Li, Z.; Chen, P.; Lu, W.; Yao, T. Raman study of gap mode and lattice disorder effect in InN films prepared by plasma-assisted molecular beam epitaxy. Acta Mater. 2007, 55, 183-187. [CrossRef] 